\title{
Dynamic Analysis of the Tilted Furuta Pendulum
}

\author{
Ali Wadi ${ }^{1, a}$, Jin-Hyuk Lee ${ }^{1}$ and Lotfi Romdhane ${ }^{1, a}$ \\ ${ }^{1}$ MCE-College of Engineering, American University of Sharjah, Sharjah, UAE
}

\begin{abstract}
This paper deals with the dynamic analysis of a tilted passive Furuta pendulum. The pendulum is tilted to ensure the existence of a stable equilibrium position. An analytical and ADAMS models are developed. These models take into account the full inertia of the pendulum and the viscous and dry friction in the joints. The only external force applied to the model is the weight. An experimental setup is used to validate the obtained models. The simulated time response of the system is compared to the experimental one. A good agreement between the two results is obtained. A frequency analysis of the response is then performed, which showed the existence of different harmonics depending on the initial angular position. Some differences are noticed, however, between the experimental results and those obtained through the simulation. These differences could be attributed to the chosen values of the viscous and dry friction parameters. A future work is underway, which uses this model to identify the friction parameters of the joints.
\end{abstract}

\section{Introduction}

The Furuta pendulum (also known as a rotary inverted pendulum) is an under-actuated system, which is formed by two bodies in series. The first one is rotating around a vertical axis followed by a pendulum rotating around a horizontal axis. It was first proposed by Furuta [1] mainly to test different control laws[2]. This mechanism is a popular one that can be found in most control laboratories. The Furuta pendulum is mainly used as a testbed of nonlinear control strategies or as an educational apparatus. Most of the works in the literature used the Furuta pendulum to illustrate their proposed control law [1]. However, most of these works did not elaborate on the dynamic model of the Furuta pendulum and they limited their work to a simplified model [3].

Few papers dealt with the modeling of the Furuta pendulum. These works highlighted the complexity of the dynamic model and the necessity to simplify based on several assumptions. The authors of $[1,4]$ proposed a linearized model based on a small angle assumption. Others neglected one or more of the cross coupling terms relating the two rotations [1]. The authors of $[2,4]$ showed, through simulations, that this assumption is not acceptable and it could have an important effect on the dynamic response. To the best knowledge of the authors, there is no work in the literature that carries time-extended experimental tests and validates the results against the deduced model. The authors of derived a significantly simplified system and carried a brief 3 -second test. All the experimental works on the Furuta pendulum are directed at testing various control strategies [5]. The dynamics of the system is often overlooked or linearized. However, the knowledge of the dynamic behavior of a

\footnotetext{
${ }^{a}$ Corresponding Author: ali.wadi@alumni.aus.edu
} 
system is highly desirable to design an advanced controller for the given nonlinear system, no matter how robust the controller is. This work presents an analytical model, which is validated through experimentation.

When the Furuta pendulum is used in a passive mode, the gravity, which is the only external load, actuates only the second joint. Therefore, the dynamic modeling of the system without an input torque, is incomplete and could not be validated through experimentation. This fact could explain the reason of the nonexistence in the literature of dynamic models of passively actuated Furuta pendulum. However, a dynamic model of the passive system could capture the physics of the system without the perturbation of the input torque. In this paper, to actuate both joints in the passive mode, we propose to tilt Furuta pendulum. In this case, the Furuta pendulum has a stable equilibrium position, defined by the gravity, and the oscillations of the pendulum around this equilibrium could be studied, analytically and experimentally. Indeed, the model shows that there is an equilibrium position around which the system will oscillate when one of the joints is moved from this position. The developed model includes the effect of the viscous and dry friction. An analytical model along with a model under ADAMS are derived. These models are then compared to the experimental results. The experimental setup uses a commercial Furuta pendulum manufactured by Quanser Inc [6]. A set of experiments were conduct with different initial positions of the pendulum. The simulation results are then compared to the experimental ones, in order to validate the model.

The rest of the paper is organized as follows: after the introduction, Section II introduces the developed models under MATLAB and ADAMS. The comparison of the results, along with a discussion, is presented in Section III. Some concluding remarks and future work are presented in Section IV.

\section{Modeling of the system}

\subsection{The Furuta Pendulum}

Figure 1 shows the Furuta pendulum in its tilted position. The angle $\phi$ is taken around the fixed y-axis. The obtained tilted reference frame is $\mathrm{x}_{0}-\mathrm{y}_{0}-\mathrm{z}_{0}$, where $\mathrm{z}_{0}$ is now the axis of the first joint between the ground and the arm (1). The joint is disconnected from the existing motor and the pendulum is subjected only to its weight. Figure 2 shows the schematic of the Furuta pendulum in its tilted position. The arm is rotating around the fixed axis $\mathrm{z}_{0}$ by an angle $\theta_{0}$ and the pendulum is rotating around the moving axis $\mathrm{x}_{1}$ by an angle $\theta_{1}$. The different parameters of the two links are defined in Table 1 , whereas those defining the joints are listed in Table 2. All these values are obtained from the manufacturer of the system [6], except for the dry friction coefficient. Several values were tested for the friction and the ones listed in Table 2 are the ones that gave the best results. It is worth noting that the ADAMS model is limited to the dry friction, whereas the analytical model uses both viscous and dry friction. 


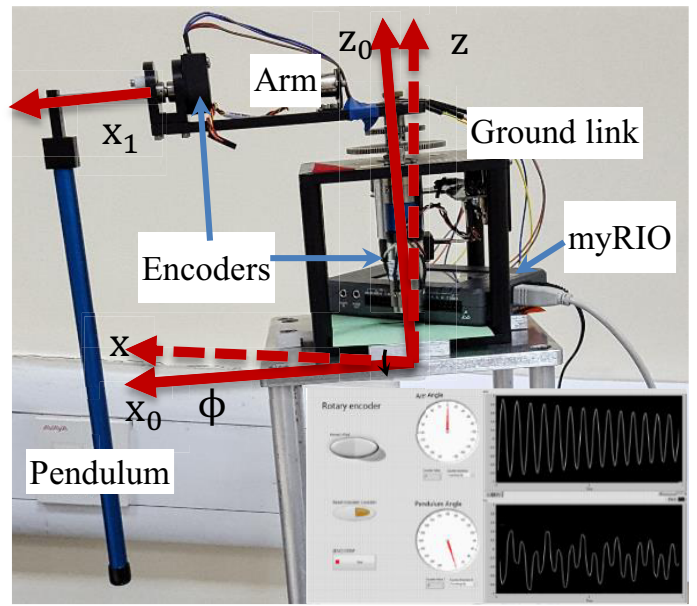

Figure 1. The tilted Furuta pendulum

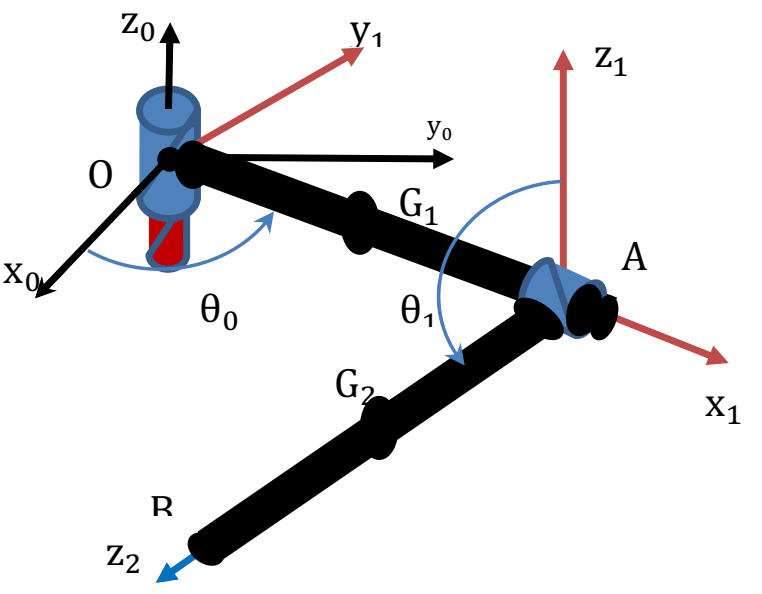

Figure 2. Schematic of the tilted Furuta pendulum.

Table 1. Parameters of the two Links

\begin{tabular}{|l|c|c|}
\hline \multicolumn{1}{|r|}{ Parameter } & Arm (1) & Pendulum (2) \\
\hline Mass $(\mathrm{Kg})$ & $m_{1}=0.370$ & $\mathrm{~m}_{2}=0.128$ \\
\hline $\begin{array}{l}\text { Moment of Inertia matrix } \\
J_{i}\end{array}$ & $j_{1 \mathrm{z}}=1.1 \times 10^{-3}$ & $\begin{array}{c}j_{2 \mathrm{xx}}=j_{2 \mathrm{yy}} \\
=5.43 \times 10^{-3} \\
\mathrm{Kg}_{2 \mathrm{zz}}=2.33 \times 10^{-6}\end{array}$ \\
\hline $\begin{array}{l}\text { Distance from pivot point } \\
\text { to center of gravity }(\mathrm{m})\end{array}$ & $l_{1}=\mathrm{OG}_{1}=0.0062$ & $l_{2}=\mathrm{AG}_{2}=0.185$ \\
\hline Total length $(\mathrm{m})$ & $\mathrm{L}_{1}=\mathrm{OA}=0.216$ & $\mathrm{~L}_{2}=\mathrm{AB}=0.316$ \\
\hline
\end{tabular}

Table 2. Parameters of the two Joints

\begin{tabular}{|r|c|c|}
\hline Parameter & Joint 1 & Joint 2 \\
\hline Radius of the shaft & $r_{1}=4$ & $r_{2}=4$ \\
\hline
\end{tabular}




\begin{tabular}{|c|c|c|}
\hline $\begin{array}{r}\text { Dry coefficient of } \\
\text { friction }\end{array}$ & $\begin{array}{c}\mu_{1}= \\
3 \times 10^{-4}\end{array}$ & $\begin{array}{c}\mu_{2}= \\
3 \times 10^{-4}\end{array}$ \\
\hline $\begin{array}{r}\text { Viscous coefficient } \\
\text { of friction } \\
\left(N . m / \frac{R a d}{s}\right)\end{array}$ & $\begin{array}{c}\eta_{1}= \\
1.3 \times 10^{-4}\end{array}$ & $\begin{array}{c}\eta_{2}= \\
1.3 \times 10^{-4}\end{array}$ \\
\hline
\end{tabular}

\subsection{Dynamic Model of the Furuta Pendulum}

\subsubsection{Analytical Model}

Based on the schematic of the Furuta pendulum shown in Figure 2, the dynamic model is elaborated. The Euler-Lagrange formalism is used to derive the equations of the motion of the system. Two generalized coordinates are required to fully describe the dynamics of the two-degrees-of-freedom system, and they are the angular displacements of the Arm and Pendulum links given by

$$
\mathbf{q}=\left[\begin{array}{l}
\theta_{0} \\
\theta_{1}
\end{array}\right]
$$

The Euler-Lagrange equation, for the $i^{\text {th }}$ generalized coordinate of the system can be written as follows:

$$
\frac{d}{d t}\left(\frac{\partial T}{\partial \dot{q}_{l}}\right)-\frac{\partial T}{\partial q_{i}}+\frac{\partial V}{\partial q_{i}}=Q_{i},(i=0,1)
$$

The kinetic energy, $\mathrm{T}$, is written as the sum of linear and rotational kinetic energies of the two links, given by

where

$$
\mathrm{T}=\sum_{\mathrm{i}=1}^{2} \frac{1}{2} \mathrm{~m}_{\mathrm{i}} \mathrm{v}_{\mathrm{Gi}}^{2}+\sum_{\mathrm{i}=1}^{2} \frac{1}{2} \omega_{\mathrm{i}-1}^{\mathrm{T}}\left(\mathrm{J}_{\mathrm{i}} \omega_{\mathrm{i}-1}\right)
$$

$\boldsymbol{\omega}_{0}=\dot{\theta}_{0} \mathbf{z}_{0}, \boldsymbol{\omega}_{1}=\dot{\theta}_{0} \mathbf{z}_{0}+\dot{\theta}_{1} \mathbf{x}_{1}$ and $\mathbf{v}_{G i}$ is the velocity of $\mathrm{G}_{i}$.

The potential energy of the gravity is written as the result following scalar product between the global vertical axis and the center of gravity position vector in the local tilted frame of reference.

$$
V=-m_{1} g\left(\mathbf{z} \cdot \mathbf{O G} \mathbf{G}_{\mathbf{1}}\right)-m_{2} g\left(\mathbf{z} \cdot \mathbf{O G}_{\mathbf{2}}\right)
$$

The generalized forces, representing the viscous and dry friction in the joints, are written as the sum of frictional torques acting on the links.

$$
Q_{j}=-\eta_{j} \dot{\theta}_{j}-\mu_{j} r_{j} F_{n j} \operatorname{sign}\left(\theta_{j}\right)
$$

where $\eta_{j}$ and $\mu_{j}$ are, respectively, the viscous and dry friction of joint $j . F_{n j}$ is the normal force in the joint $\mathrm{j}$.

The final model is given as follows:

$$
H \ddot{q}+B(q, \dot{q})+G(q)=Q
$$

where:

The generalized inertia $2 \times 2$ matrix is given by:

$$
\mathbf{H}=\left[\begin{array}{cc}
\left(\mathrm{j}_{1 \mathrm{z}}+\mathrm{m}_{1} l_{1}^{2}+\mathrm{m}_{2} \mathrm{~L}_{1}^{2}+\left(\mathrm{m}_{2} l_{2}^{2}+\mathrm{j}_{2 \mathrm{y}}\right) \sin \left(\theta_{1}\right)^{2}+\mathrm{j}_{2 \mathrm{z}} \cos \left(\theta_{2}\right)^{2}\right) & -m_{2} l_{2} L_{1} \cos \left(\theta_{1}\right) \\
-\left(m_{2} l_{2} L_{1} \cos \left(\theta_{1}\right)\right) & j_{2 x}+m_{2} l_{2}^{2}
\end{array}\right]
$$

The centrifugal and Coriolis forces are given by the following vector:

$$
\mathbf{B}(\mathbf{q}, \dot{\mathbf{q}})=\left[\begin{array}{c}
-\left(\left(m_{2} l_{2}^{2}-j_{2 y}+j_{2 x}\right) \sin \left(2 \theta_{1}\right)\right) \dot{\theta}_{0} \dot{\theta}_{1}+\left(m_{2} l_{2} L_{1} \sin \left(\theta_{1}\right)\right) \dot{\theta}_{1}^{2} \\
\left(\left(-m_{2} l_{2}^{2}-j_{2 y}+j_{2 z}\right) \sin \left(\theta_{1}\right) \cos \left(\theta_{1}\right)\right) \dot{\theta}_{0}^{2}
\end{array}\right]
$$

The effect of gravity is given by the following vector

$$
\mathbf{G}(\mathbf{q})=\left[\begin{array}{c}
\left(m_{1} g l_{1} \sin \left(\theta_{0}\right) \sin (\phi)+m_{2} g\left(L_{1} \sin \left(\theta_{0}\right)-l_{2} \sin \left(\theta_{2}\right) \cos \left(\theta_{0}\right)\right) \sin (\phi)\right) \\
\left(-m_{2} g l_{2}\left(\sin \left(\theta_{0}\right) \cos \left(\theta_{1}\right) \sin (\phi)-\sin \left(\theta_{1}\right) \cos (\phi)\right)\right)
\end{array}\right]
$$

The generalized forces due to friction are given by the following vector: 


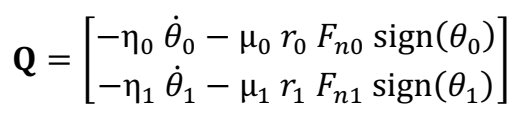

\subsubsection{ADAMS Model}

Figure 3 shows the model under the software ADAMS. The gravity vector is shown downwards. It is worth mentioning that in the ADAMS model, the gravity is tilted by the angle $\phi$ with respect to the axis of the first joint. The friction is modeled in ADAMS using its building model. The parameters used are given by the menu shown on Figure 3. Only the dry friction parameter values are changed, all the other parameters are kept as default values given by ADAMS. It is worth mentioning that the viscous friction is not included in this model.

The output of the model is the motion of the first and second joints, for different initial positions of the second joint.

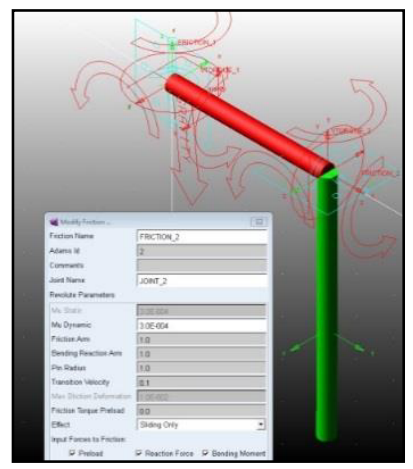

Figure 3. The ADAMS model of the tilted Furuta pendulum

\subsubsection{Experimental Setup}

The experimental setup is made of an instrumented Furuta pendulum, an acquisition system (myRIO Embedded System, National Instruments), and a computer with the LabVIEW software (Figure 1). The system is equipped by two rotary encoders (E2 Optical Kit Encoder by US Digital) to record the angular positions of the two joints as a function of time.

The sampling period is chosen to be $10 \mathrm{~ms}$ and the acquisition time covers the full motion until the pendulum stops. This time ranged from 12 to $45 \mathrm{~s}$, depending on the initial angular position of the motion.

\section{Results and Discussion}

The simulation results (analytical and ADAMS) and the experimental results are obtained for two different initial positions of the pendulum (2). These positions are chosen to be 15 degrees and 55 degrees, and they are all taken with respect to the equilibrium position, which is along the negative $\mathrm{z}$ axis. These values are chosen to have a small angle ( 15 degrees) and a large angle ( 55 degrees).

In all the experiments the pendulum starts with a zero initial angular velocity. The motion in the two joints is monitored until the system returns to the equilibrium position. The obtained results from the three cases (Analytical, ADAMS, Experimental) are the time responses of the two joint angles. For the experimental setup, the recording was repeated several times and no differences were observed between different runs having the same initial angular position. Based on this time response, the decay of the amplitude as a function of time and the frequency analysis were performed. 


\subsection{Time response}

The time response for the two cases of initial angular positions are shown in Figure 4. These results show that the pendulum has a motion similar to a simple pendulum, however the arm is animated by highly complex motion. This complexity is predicted by the analytical model, due to the coupling between the two motions and the nonlinear terms. None of the two models (analytical and ADAMS) fitted the experimental results. For the pendulum motion, the analytical model was closer to the experimental result despite the slight difference in its natural frequency, which led to a shift between the two curves after a few periods of motion. The ADAMS model had a similar problem with the frequency but also the amplitude was not predicted correctly. This difference in the two cases could not only be attributed to the high nonlinearity in the model but also to the models used in describing the viscous and dry frictions. Indeed, the analytical model used Eq. 4 along with Table 2 to describe the model of the joints. In ADAMS, the Coulomb friction parameters are shown on Figure 3. However, the model of the joints is not taking into account the viscous friction.
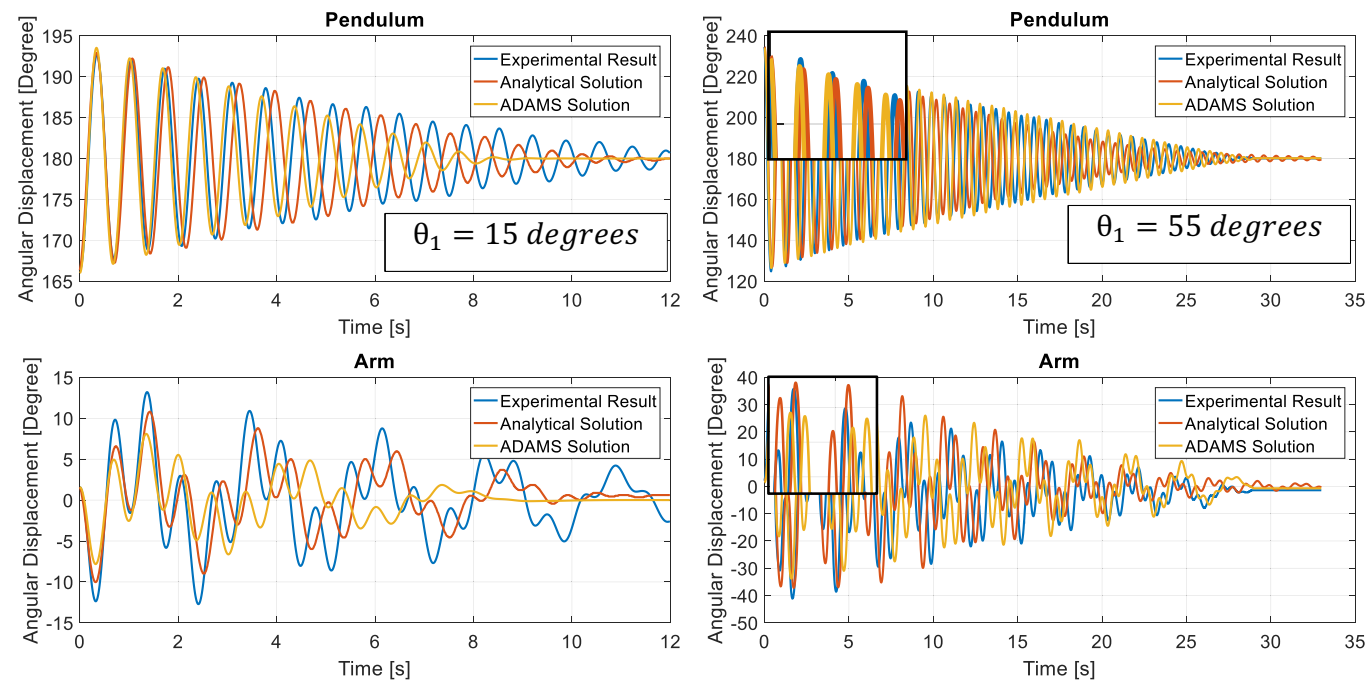

Figure 4. Time response of the arm and pendulum

\subsection{Frequency Response}

The frequency analyses of the responses are shown in Figure 5 for the arm and the pendulum. These figures show the frequency responses for three initial angles, i.e., 15, 55 and 90 degrees. One can notice that the analytical model predicted the results better than the ADAMS model in all cases. Another important result from these plots is the number of harmonics, in the response of the arm, that changes from one, in the case of $\theta_{1}=15$ degrees to five in the case of $\theta_{1}=90$ degrees. This is result shows again the high nonlinearity of the model. At $\theta_{1}=15$ degrees, there is only one dominant frequency at around $1.5 \mathrm{~Hz}$. Lower frequencies cannot be detected due to the high signal to noise ratio. For the case of $\theta_{1}=55$ degrees, four harmonics, 0.4-1.2-3.6-5.8 Hz, are detected for the arm and five harmonics at $\theta_{1}=90$ degrees, i.e., 0.4-1-3-5-7 Hz. For the pendulum, the response is similar in all cases, except at $\theta_{1}=90$ degrees, where a second harmonic at $3 \mathrm{~Hz}$ starts to appear. This type of behavior shows the high nonlinearity of the system. Despite this complexity, the proposed model succeeded in capturing the physics of the system in all three cases. Some differences persist which could be attributed to the values used for the friction parameters. 

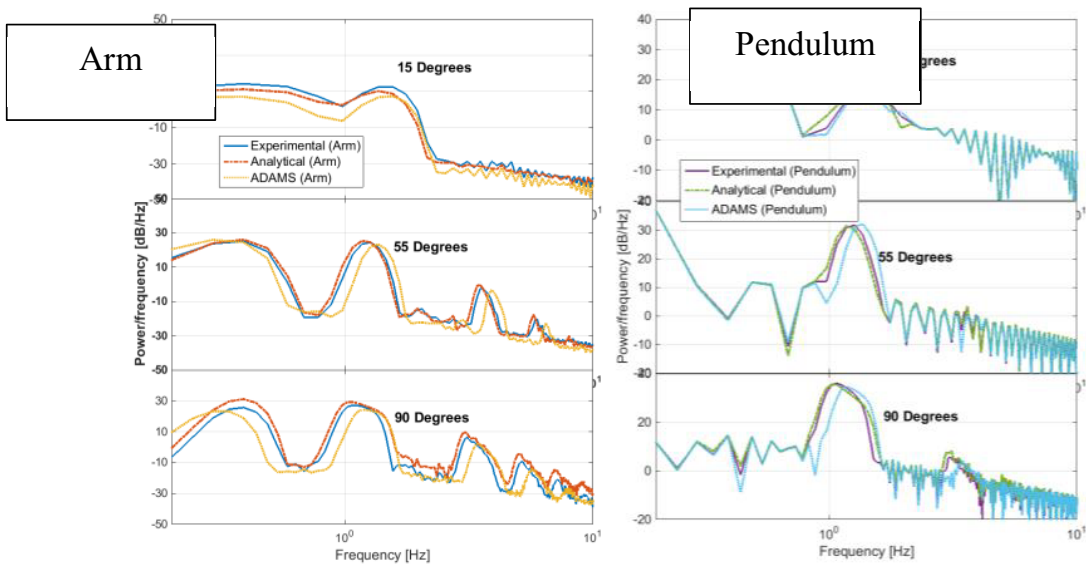

Figure 5. Frequency response for the arm and pendulum at different initial angles

\section{Conclusion}

This paper presented a dynamic analysis of a tilted Furuta pendulum. The pendulum was tilted to ensure the existence of a stable equilibrium position. A complete analytical model was derived where all the full inertia of the pendulum was taken into account. ADAMS was also used to simulate the system. The obtained results showed that the developed analytical model gave results that are closer to the experimental ones and hence predicted better the real dynamic behavior of the system.

A frequency analysis was performed and it showed that the system is highly nonlinear. Different harmonics were detected depending on the initial angular position. The difference between the simulation results and the experimental ones could be attributed to the values of the viscous and dry friction parameters and the model chosen for these frictions.

\section{Acknowledgment}

This work was financed by the American University of Sharjah under the grant FRG15-40.

\section{References}

1. K. Furuta, M. Yamakita and S. Kobayashi, "Swing-up control of inverted pendulum using pseudo-state feedback", Proceedings of the Institution of Mechanical Engineers, Part I: Journal of Systems and Control Engineering 1991-1996 (vols 205-210), 206, no. 49, pp. 263-269, 1992.

2. I. S. Diniz, D. Colón, R. Carrion, R. Aguera, L. Góes, "The Rotary Inverted Pendulum: Modeling, Simulation and Control Aided by Computer: CAD-CAE", 22nd International Congress of Mechanical Engineering, Ribeirão Preto, Brazil, 2013.

3. S. Jadlovský and J. Sarnovský, "Modelling of Classical and Rotary Inverted Pendulum Systems A Generalized Approach," Journal of Electrical Engineering, 64, no. 1, Jan. 2013.

4. B. S. Cazzolato and Z. Prime, "On the Dynamics of the Furuta Pendulum," Journal of Control Science and Engineering, 2011, pp. 1-8, 2011.

5. L. T. Aguilar, "Identification based generation of self-excited oscillations for underactuated mechanical systems via two-relay algorithm," 2008 International Workshop on Variable Structure Systems, Turkey, doi:10.1109/VSS.2008.4570680.

6. "Quanser - Rotary Inverted Pendulum", Quanser.com, 2016. [Online]. Available: http://www.quanser.com/products/rotary_pendulum. [Accessed: 15-Jul- 2016]. 